\title{
The formation of brown dwarfs
}

\author{
Ant Whitworth, ${ }^{1}$ Dimitri Stamatellos, ${ }^{1}$ Steffi Walch, ${ }^{1}$ Murat Kaplan, ${ }^{1}$ \\ Simon Goodwin, ${ }^{2}$ David Hubber ${ }^{2}$ and Richard Parker ${ }^{2}$ \\ ${ }^{1}$ School of Physics \& Astronomy, Cardiff University, Cardiff CF24 3AA, UK \\ email: ant@astro.cf.ac.uk \\ ${ }^{2}$ Department of Physics \& Astronomy, University of Sheffield, Sheffield S3 7RH, UK
}

\begin{abstract}
We argue that brown dwarfs (BDs) and planemos form by the same mechanisms as low-mass hydrogen-burning stars, but that as one moves to lower and lower masses, an increasing fraction of these objects is formed by fragmentation of the outer parts $(R \gtrsim 100 \mathrm{AU})$ of protostellar accretion discs around more massive primary protostars, which in turn formed in their own very-low-mass prestellar cores. Numerical simulations of disc fragmentation with realistic thermodynamics show that low-mass objects are readily formed by fragmentation of short-lived massive, extended protostellar accretion discs. Such objects tend subsequently to be liberated into the field at low speed, due to mutual interactions with the primary protostar. Many $(\sim 20 \%)$ are in low-mass $\left(M_{1}+M_{2}<0.2 \mathrm{M}_{\odot}\right)$ binary systems with semi-major axes $a \sim 1$ to $2 \mathrm{AU}$ or $\sim 200 \mathrm{AU}$ and mass ratios $q \equiv M_{2} / M_{1} \gtrsim 0.7$. Most of the brown dwarfs have sufficiently large attendant discs to sustain accretion and outflows. Most of the BDs that remain bound to the primary protostar have wide orbits (i.e., there is a BD desert), and these BDs also have a significantly higher probability of being in a BD/BD binary system than do the brown dwarfs that are liberated into the field (just as observed). In this picture, the multiplicity statistics and velocity dispersion of brown dwarfs are largely determined by the eigen evolution of a small- $N$ system, born from a single prestellar core, rather than the larger-scale dynamics of the parent cluster. Consequently, many of the statistical properties of brown dwarfs should not differ very much from one star-formation region to another.
\end{abstract}

Keywords. accretion, accretion disks, gravitation, hydrodynamics, radiative transfer, stellar dynamics, binaries: general, stars: formation, stars: low-mass, brown dwarfs

\section{Introduction}

The appearance of the Universe we live in, and the environment in which we ourselves have prospered, are both strongly conditioned by the fact that almost all seriously dense (say, $\rho \gtrsim 1 \mathrm{~g} \mathrm{~cm}^{-3}$ ) baryonic matter in the Universe is in objects with a rather small logarithmic range of mass (say, 0.01 to $100 \mathrm{M}_{\odot}$ ), i.e., stars. The study of star formation is largely concerned with understanding why this is so. There are two main approaches to answering this question. One approach argues that the thermodynamics of the interstellar medium delivers a more or less universal mean Jeans mass $\sim 0.3 \mathrm{M}_{\odot}$, and then the range of stellar masses simply reflects the variances of all factors determining the final stellar mass in a particular location. This is the basis of turbulence theories. The second approach argues that it becomes increasingly hard to form stars as one goes to higher masses (because self-gravitating gas has to go through ever more complicated contortions to get past radiation pressure) or lower masses (because opacity makes it increasingly hard for self-gravitating gas to lose entropy on a dynamical timescale), so that there is naturally a concentration of masses between these limits. The full solution may actually involve all three elements (mean Jeans mass, radiation pressure and opacity). Here we will be most concerned with understanding the formation of low-mass stars, i.e., star formation close 
to the opacity limit, and what constraints can be inferred from the observed properties of such stars.

In the context of star formation, it is appropriate to describe brown dwarfs (and planemos) as stars. This is because all available evidence (e.g., Luhman et al. 2007) suggests that brown dwarfs form just like low-mass hydrogen-burning stars, i.e., by gravitational instability, on a dynamical timescale $\dagger$, normally (possibly always) in clusters, and with an initially uniform elemental composition (the same as the elemental composition of the interstellar medium out of which they condense). There is no compelling evidence for a discontinuity in stellar statistical properties across the hydrogen-burning limit at $\mathrm{M}_{\mathrm{H}} \simeq 0.075 \pm 0.005 \mathrm{M}_{\odot}$, at least, not in those properties that might be expected to reflect the formation process of the objects concerned (as distinct from the nuclear processes which contribute later to their intrinsic luminosity and long-term evolution). With this definition of a star, the opacity limit, which is variously estimated to be between 0.003 and $0.007 \mathrm{M}_{\odot}$, is the minimum mass for star formation. (It makes no sense to call it the minimum mass for planemo formation, because it evidently is not!)

In contrast, planets form by accumulation of a rocky core, on a much longer timescale, $\gtrsim 10^{7} \mathrm{yr}$, with subsequent acquisition of a gaseous envelope if the circumstances allow this, and with an initially fractionated elemental composition. With these definitions of stars and planets, based on their formation mechanisms, there is probably an interval in the mass range 0.003 to $0.010 \mathrm{M}_{\odot}$ which hosts both stars and planets. It may be quite hard to distinguish the two without information on their internal compositions.

In Section 2, we describe briefly the different formation mechanisms that have been proposed for brown dwarfs. In Section 3, we rehearse the observational constraints on brown dwarf formation. In Section 4, we explore the possibility that brown dwarfs form by disc fragmentation, and show that this mechanism reproduces the observed statistical properties of brown dwarfs rather well. In Section 5, we summarise our conclusions.

\section{How do brown dwarfs form?}

Turbulence: Brown dwarfs and low-mass hydrogen-burning stars might form by turbulent fragmentation (e.g., Klessen \& Burkert 2002; Padoan \& Nordlund 2002; Hennebelle \& Chabrier 2008). In this picture, it is envisaged that turbulent energy is injected into the interstellar medium on large scales (possibly by the gravitational and/or thermal instabilities involved in the formation of molecular clouds, or by galactic shear), and then cascades to smaller and denser scales, until it creates self-gravitating cores which condense to form stars. Allowing for the fact that a single pre-stellar core is not expected to put all its mass into a single star, this requires that occasionally, not very often, turbulence can deliver self-gravitating cores with masses $\lesssim 0.01 \mathrm{M}_{\odot}$. This has not yet been demonstrated to happen in a numerical simulation. As presently developed, the theory also has the following outstanding issues. (i) It seems to require some fine tuning, perhaps by as yet unidentified thermodynamic processes, to keep the ratio of brown dwarfs to hydrogen-burning stars roughly constant, as observed across widely differing star-formation regions. (ii) The mechanism seems to imply that more massive stars form in less dense, more extended cores, and hence in the outer parts of clusters. This may be difficult to reconcile with the growing evidence for primordial mass segregation. (iii) The mechanism does not yet make any firm predicitons about binary statistics.

$\dagger$ We note that the dynamical timescale for low-mass stars condensing out of gas with isothermal sound speed $c \simeq 0.2 \mathrm{~km} \mathrm{~s}^{-1}$ (i.e., $\mathrm{H}_{2}$ at $\sim 10 \mathrm{~K}$ ) is given by $t_{\mathrm{dyn}} \simeq G M_{\star} / c^{3} \simeq$ 600 years $\left(M_{\star} / \mathrm{M}_{\mathrm{Jupiter}}\right)$ and is therefore very short for low-mass objects. 
Dynamical fragmentation and ejection: Brown dwarfs and low-mass hydrogen-burning stars might also form by dynamical fragmentation and ejection. This mechanism starts with a pre-stellar core (perhaps formed by turbulent fragmentation), but the core is sufficiently massive and turbulent that it collapses and undergoes dynamical fragmentation, thereby producing a small ensemble of protostellar embryos at the centre of an infalling envelope. Some of these protostellar embryos are then ejected from the core by dynamical interactions amongst themselves, and consequently never acquire sufficient mass to ignite hydrogen-burning. Those that remain in the core compete to accrete the remaining mass, and become hydrogen-burning stars with a range of masses. This mechanism was first proposed by Reipurth \& Clarke (2000), and has been demonstrated on the basis of numerical simulations by Bate et al. (2003) and Goodwin et al. (2004). It has two main problems. First the brown dwarfs tend to be ejected with rather large velocities, and therefore there should be a diaspora of brown dwarfs visible round young clusters. Despite concerted observational efforts to detect it, such a diaspora has not been observed. Second, this mechanism does not seem either to produce the tight BD/BD binary systems in which about $\sim 20 \%$ of brown dwarfs are found, or to explain the brown-dwarf desert (the paucity of close brown-dwarf companions to Sun-like stars).

Photo-erosion of pre-existing cores: Brown dwarfs and low-mass hydrogen-burning stars might also form where a pre-existing core is overrun by an HiI region, as first proposed by Hester (1996). In this case, there is a competition between core collapse and the boiling off of its outer layers by ionisation. Whitworth \& Zinnecker (2004) have shown that this is a very robust mechanism, in the sense that brown dwarfs are produced for a wide range of likely initial conditions. Moreover, evaporating gaseous globules (EGGs) are seen in M16, so the mechanism evidently operates. However, it is a very inefficient mechanism. It requires quite a massive core to make a single brown dwarf. In addition, it can only operate in clusters that produce OB stars, so it cannot explain the brown dwarfs observed in lower-mass star-formation regions like Taurus. Finally, it makes no predictions about the binary statistics of brown dwarfs. It is - at best - a viable way to produce some single brown dwarfs in star-formation regions like the Orion Nebula Cluster.

Disc fragmentation and liberation: In the remainder of this contribution, we shall promote the idea that a large fraction of brown dwarfs and some low-mass hydrogen-burning stars form by fragmentation of accretion discs around more massive primary protostars. In this picture, the low-angular-momentum material in a pre-stellar core collapses first to form the primary protostar (hereafter the ur star). Much of the remaining material in the core then forms an accretion disc round the ur star. Once this disc becomes sufficiently massive $\left(M_{\text {disc }} \gtrsim 0.3 M_{\star}\right)$ and extended $\left(R \gtrsim 100 \mathrm{AU}\left(M_{\star} / \mathrm{M}_{\odot}\right)^{1 / 3}\right)$, it fragments to form lower-mass secondaries in its outer parts, primarily brown dwarfs and very-low-mass hydrogen-burning stars, but also a few planemos. Gravitational interactions between the secondaries and the ur star then liberate most of the secondaries, but a few remain bound to the ur star. More specifically, we argue that, as one proceeds to lower masses, from low-mass hydrogen-burning stars through brown dwarfs to planemos, an increasing fraction of stars is formed as secondaries by disc fragmentation, rather than being formed as primaries by the monolithic collapse of a core, or by dynamical fragmentation during the collapse of a core. By means of numerical simulations, we show that this formation mechanism seems to be able to deliver all observed statistical properties of brown dwarfs. One advantage of this model is that most statistical properties of brown dwarfs and lowmass hydrogen-burning stars are determined by the eigen evolution of a small- $N$ system formed within a single core, so they are largely independent of environmental factors, 
or of the overall dynamics of the parent cluster. André et al. (2007) recently presented evidence that the prestellar cores in Ophiuchus are sufficiently far apart, and move sufficiently slowly (relative to one another), that their internal evolution will occur more or less in isolation.

\section{Constraints on brown dwarf formation}

The stellar initial mass function (IMF): The distribution of stellar masses - at least for contemporary, local star formation - is approximately lognormal (e.g., Kroupa 2001; Chabrier 2003), and similar in shape to the observed prestellar-core mass function. However, the core-mass function peaks at a mass about three times higher than the stellar IMF. Thus, to reproduce the masses and binary statistics of stars, a typical prestellar core must convert between 30 and $60 \%$ of its mass into between two and five stars. Since (a) the core-mass function is not significantly narrower than the stellar IMF and (b) the masses of stars produced by a single core are not identical, the mapping from core-mass function to stellar IMF cannot be self-similar. Rather, lower-mass cores must, on average, produce fewer stars and/or convert a higher fraction of their mass into stars. Hence, the decline in the stellar IMF at low masses reflects both the decline in the core-mass function at low masses, and the difficulty of producing low-mass secondaries in such cores. In fact, in a low-mass, low-turbulence core, disc fragmentation is particularly effective at producing secondaries, because material can be parked in a disc, losing entropy, whilst it slowly builds up towards gravitational instability.

Accretion and outflows: A significant fraction of young brown dwarfs show evidence of accretion discs, magnetospheric accretion, bipolar outflows, etc. The inference is that their formation is simply a scaled-down version of the formation of higher-mass stars. For example, mass-accretion rates appear to scale approximately as the stellar mass squared, $\dot{M}_{\star} \propto M_{\star}^{2}$, for both hydrogen-burning stars and brown dwarfs. However, we should be mindful that these observations simply require young brown dwarfs to be attended by significant accretion discs, i.e., that they did not form from material with negligible angular momentum, and that they have not suffered a sufficiently violent perturbation to separate them from their accretion discs. A brown dwarf of mass $m_{\mathrm{bd}}$, which is in orbit round an ur star of mass $M_{\mathrm{ur}}$ at radius $R_{\text {orbit }}$, will, if subsequently ejected by dynamical interactions with other stars, retain a disc of radius $r_{\mathrm{disc}} \sim R_{\text {orbit }}\left(m_{\mathrm{bd}} / M_{\mathrm{ur}}\right)^{1 / 2}$. We will show that brown dwarfs formed by disc fragmentation are formed at such large distances from the ur star that they retain discs several tens of AU in extent, in accordance with observations.

Maps and SEDs: If brown dwarfs are formed by disc fragmentation, one might hope to see evidence for massive extended discs in continuum maps and SEDs of Class 0 and I protostars. The problem here is that the process of disc fragmentation is very fast, and the fragmenting disc is likely to be deeply embedded in the core envelope. Therefore, the disc is probably already largely dissipated by the time that the protostar reaches the Class I phase.

Spatial and velocity distribution in clusters: The distributions of brown dwarfs in young and evolved clusters should hold clues to the way in which brown dwarfs form. However, these clues have proved hard to decipher. Setting aside the issue of selection effects (brown dwarfs become increasingly hard to detect as they age and fade), the problem is that one has to disentangle primordial mass segregation (for example, any tendency that brown dwarfs might have to form preferentially in the outer parts of a cluster), from subsequent segregation due to systematic differences in the velocity dispersions of stars of different mass. This second effect works on three levels, the first being the 
eigen evolution of the stars formed in a single core (which determines the local velocity dispersion of the individual stars relative to the centre of mass of the core), the second being the overall dynamics of the cluster (which determines the velocity dispersion of the individual cores relative to one another, and may involve violent relaxation), and the third being dynamical mass segregation (more quasistatic relaxation driven by equipartition of energy).

Binary statistics: Arguably the most discriminating constraints on brown-dwarf formation derive from the binary statistics of brown dwarfs. As one looks at stars of decreasing mass, the probability of their being the primary in a binary system decreases [for brown dwarfs, $\left.f_{\text {bin }} \sim 0.20 \pm 0.05\right]$, and - for those that are primaries - the mean semi-major axis decreases [for brown dwarfs, $\bar{a} \sim 5 \pm 2 \mathrm{AU}$ ], the (logarithmic) spread of semi-major axes decreases [for brown dwarfs, $\sigma_{\log _{10}(a)} \sim 0.4 \pm 0.1$ ] and the mean mass ratio increases [for brown dwarfs, $\bar{q} \sim 0.8 \pm 0.1]$. Two further aspects are remarkable. (i) When Sun-like stars have brown-dwarf companions, the semi-major axis is normally large, say, $a \sim 200 \mathrm{AU}$. Close systems are very rare (this is termed the Brown Dwarf Desert; e.g., Burgasser et al. 2007). (ii) Those brown dwarfs that are companions to Sun-like stars (orbiting at $a \sim 200 \mathrm{AU})$ are much more likely to be in a close $(a \sim 5 \mathrm{AU})$ binary system with another brown dwarf than brown dwarfs in the field (Burgasser et al. 2005).

\section{Disc fragmentation}

Suppose that there exists a Keplerian disc with surface density $\Sigma(R)$, sound speed $a(R)$ and orbital angular speed $\Omega(R)=\left(G M_{\mathrm{ur}} / R^{3}\right)^{1 / 2}$, where $r$ is the radial distance from the central ur star. Now consider a small circular proto-fragment with radius $r$, and hence mass $m=\pi r^{2} \Sigma(R)$. The equation controlling its radial excursions is

$$
\begin{aligned}
\ddot{r} & \simeq-\frac{G m}{r^{2}}+\frac{1}{\rho} \frac{\mathrm{d} P}{\mathrm{~d} r}+\frac{\Omega^{2}(R) r}{4} \\
& \simeq-\pi G \Sigma(R)+\frac{a^{2}(R)}{r}+\frac{\Omega^{2}(R) r}{4} .
\end{aligned}
$$

Hence, the timescale on which the proto-fragment condenses out is given by

$$
t_{\text {cond }} \simeq\left(\frac{r}{-\ddot{r}}\right)^{1 / 2} \simeq\left(\frac{\pi G \Sigma(R)}{r}-\frac{a^{2}(R)}{r^{2}}-\frac{\Omega^{2}(R)}{4}\right)^{-1 / 2}
$$

and the fastest-condensing proto-fragment has radius and growth time given by

$$
\begin{aligned}
& r_{\text {fast }} \simeq \frac{2 a^{2}(R)}{\pi G \Sigma(R)}, \\
& t_{\text {fast }} \simeq 2\left(\left(\frac{\pi G \Sigma(R)}{a(R)}\right)^{2}-\Omega^{2}(R)\right)^{-1 / 2} .
\end{aligned}
$$

Evidently, the growth time is only real if

$$
\Sigma(R) \gtrsim \frac{a(R) \Omega(R)}{\pi G}
$$

which is the condition for the disc to be unstable to gravitational fragmentation (Toomre 1964; Goldreich \& Lynden-Bell 1965). In an equilibrium disc that is too massive to be Keplerian, $\Omega$ should be replaced by the epicyclic frequency, and in a nonequilibrium disc by twice the local vorticity, but otherwise the condition takes the same form. 
Gammie (2001) has shown that such a disc will only fragment if a proto-fragment can cool on a dynamical timescale, otherwise the fragment undergoes an adiabatic bounce and is sheared apart. For a marginally unstable disc, the dynamical timescale is

$$
t_{\mathrm{dyn}} \simeq \frac{2 a(R)}{\pi G \Sigma(R)} .
$$

The cooling time is given by

$$
t_{\mathrm{cool}} \simeq \frac{\Sigma(R) a^{2}(R)}{2 \sigma_{\mathrm{sb}} T^{4}(R) / \Sigma(R) \bar{\kappa}_{\mathrm{R}}(R)}=\frac{15 c^{2} h^{3} \Sigma^{2}(R) \bar{\kappa}_{\mathrm{R}}(R)}{2^{2} \pi^{5} \bar{m}^{4} a^{6}(R)} .
$$

In the first expression, the numerator, $\Sigma a^{2}$, is the thermal energy per unit area and the denominator, $2 \sigma_{\mathrm{sb}} T^{4}(R) / \Sigma(R) \bar{\kappa}_{\mathrm{R}}(R)$, is the blackbody flux from the two sides of the disc, divided by the optical depth, $\tau=\Sigma \bar{\kappa}_{\mathrm{R}}$. Here, $\bar{\kappa}_{\mathrm{R}}$ is the Rosseland mean opacity and we are assuming that the disc is optically thick (which can be confirmed retrospectively). To obtain the second expression, we have substituted $\sigma_{\mathrm{sb}} T^{4}=2 \pi^{5} \bar{m}^{4} a^{8} / 15 c^{2} h^{3}$, where $\bar{m}$ is the mean gas-particle mass $\left(\sim 4 \times 10^{-24} \mathrm{~g}\right.$ for molecular gas). Combining Equations (4.7) and (4.8), Gammie's condition, $t_{\text {cool }}<t_{\mathrm{dyn}}$, reduces to

$$
\Sigma(R) \lesssim\left(\frac{2^{3} \pi^{4} \bar{m}^{4} a^{7}(R)}{15 G c^{2} h^{3} \bar{\kappa}_{\mathrm{R}}(R)}\right)^{1 / 3}
$$

We shall assume that in cool parts of the disc, the opacity is dominated by ice-coated grains with emission efficiency proportional to frequency squared. Hence, the Rosseland mean opacity is proportional to the temperature squared, or the sound speed to the fourth power. For standard interstellar dust, this gives $\bar{\kappa}_{\mathrm{R}} \simeq \kappa_{\mathrm{O}} a^{4}$ with $\kappa_{\mathrm{O}} \simeq 3 \times$ $10^{-19} \mathrm{~s}^{4} \mathrm{~cm}^{-2} \mathrm{~g}^{-1}$. With this expression for $\bar{\kappa}_{\mathrm{R}}$, Eqn. (4.9) becomes

$$
\Sigma(R) \lesssim\left(\frac{2^{3} \pi^{4} \bar{m}^{4}}{15 G c^{2} h^{3} \kappa_{\mathrm{O}}}\right)^{1 / 3} a(R)
$$

Since the lower limit on $\Sigma$ in Equation (4.6) and the upper limit in Equation (4.10) are both linear in $a$, when we combine them, the dependence on $a$ drops out, and we are left with a constraint on $\Omega$ :

$$
\Omega(R) \lesssim\left(\frac{2^{3} \pi^{7} G^{3} \bar{m}^{4}}{15 c^{2} h^{3} \kappa_{\mathrm{O}}}\right)^{1 / 3}
$$

or equivalently (for a Keplerian disc)

$$
R \gtrsim R_{\min }\left(M_{\mathrm{ur}}\right) \simeq\left(\frac{15^{2} c^{4} h^{6} \kappa_{\mathrm{O}}^{2} M_{\mathrm{ur}}^{3}}{2^{6} \pi^{14} G \bar{m}^{8}}\right)^{1 / 9} \simeq 100 \mathrm{AU}\left(\frac{M_{\mathrm{ur}}}{\mathrm{M}_{\odot}}\right)^{1 / 3}
$$

This is a rather robust explanation for the brown-dwarf desert. Only the outer parts of a circumstellar disc can be both massive enough, and at the same time able to cool fast enough, to fragment. Moreover, the masses of the secondaries that form there are typically in the range 0.003 to $0.1 \mathrm{M}_{\odot}$ (see Whitworth \& Stamatellos 2006 for details). Parenthetically, we note that the dependence on the dust emissivity is very weak: first, $R_{\min } \propto \kappa_{\mathrm{O}}^{2 / 9}$; second, even if the dependence on $a$ does not cancel out exactly (i.e., $\bar{\kappa}_{\mathrm{R}}$ is not $\propto a^{4}$ ), the dependence of $a$ on $R$ is so weak compared with that of $\Omega$ (typically, $a \propto R^{-1 / 4}$ as opposed to $\Omega \propto R^{-3 / 2}$ ) that the result is unlikely to be changed by much. Thus, even if the dust properties in a protostellar disc evolve away from those in the general interstellar medium, this is unlikely to have a large effect. 
We note that to form a disc of this extent requires the material trying to accrete onto the ur star to have angular momentum

$$
h \gtrsim h_{\mathrm{min}} \simeq 3 \times 10^{20} \mathrm{~cm}^{2} \mathrm{~s}^{-1}\left(\frac{M_{\mathrm{ur}}}{\mathrm{M}_{\odot}}\right)^{2 / 3} \equiv\left(0.1 \mathrm{~km} \mathrm{~s}^{-1}\right)(0.01 \mathrm{pc})\left(\frac{M_{\mathrm{ur}}}{\mathrm{M}_{\odot}}\right)^{2 / 3} .
$$

Alternatively, the original core must have

$$
\beta \equiv \frac{U_{\text {rot }}}{\left|U_{\text {grav }}\right|} \gtrsim 0.005\left(\frac{M_{\mathrm{ur}}}{\mathrm{M}_{\odot}}\right)^{4 / 3}
$$

These are very modest requirements compared with the observed properties of prestellar cores.

We have simulated disc fragmentation using smoothed-particle hydrodynamics, solving the energy equation with a scheme that treats realistically the equation of state of protostellar gas and the transport of cooling radiation against dust opacity (for details see Stamatellos et al. 2007), and introducing sink particles only at very high densities ( $\gtrsim 10^{-3} \mathrm{~g} \mathrm{~cm}^{-3}$; Stamatellos et al. 2007; Stamatellos \& Whitworth 2009a,b). We find that fragmentation is limited to the outer parts of a protostellar disc, and forms multiple secondaries with masses in the range 0.003 to $0.200 \mathrm{M}_{\odot}$. Dynamical interactions amongst these secondaries have two main effects. First, a significant fraction end up in tight $(a \sim 1$ to $2 \mathrm{AU})$, low-mass $\left(M_{1}+M_{2} \lesssim 0.2 \mathrm{M}_{\odot}\right)$ binary systems. Second, the higher-mass secondaries tend to be scattered or migrate into tighter orbits (relative to the ur star), whilst the lower-mass secondaries tend to be liberated into the field. And the intermediate-mass secondaries remain at large distances from the ur star. Thus, the properties of the brown-dwarf desert are reproduced. In addition, (i) both bound and
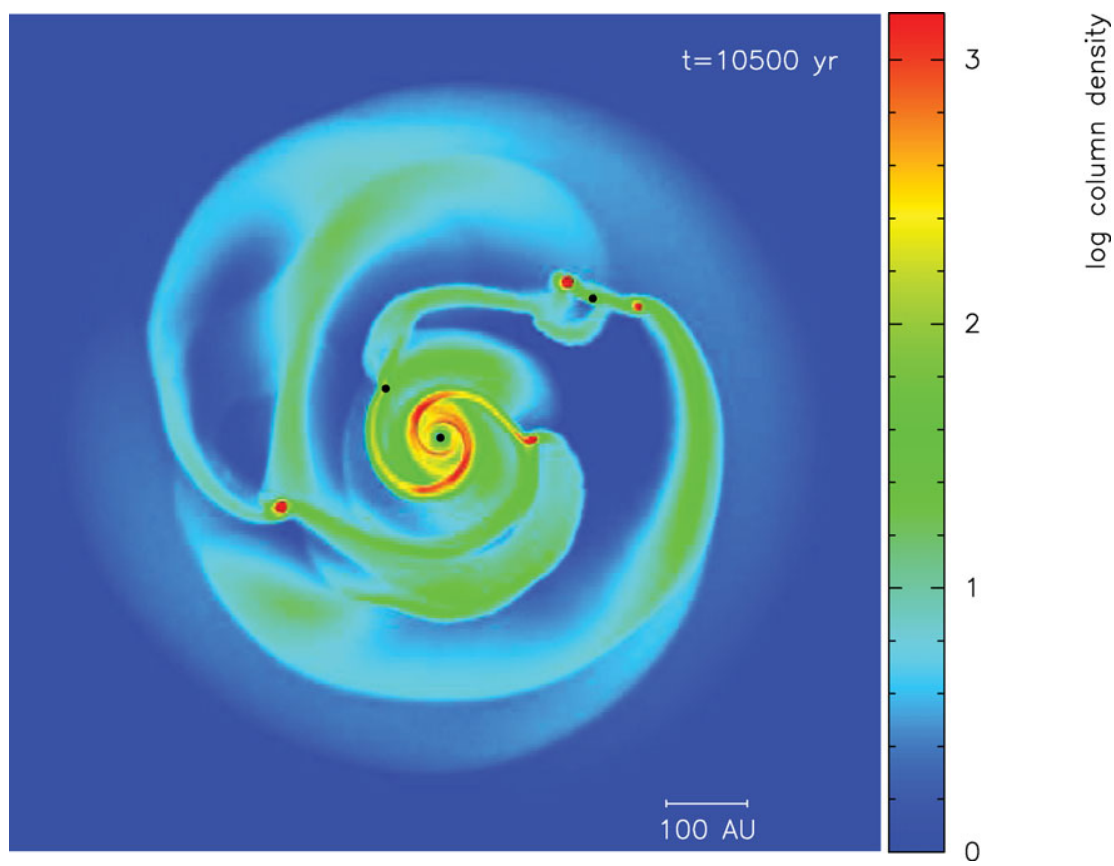

Figure 1. Simulation of the fragmentation of an $0.7 \mathrm{M}_{\odot}$ disc round an $0.7 \mathrm{M}_{\odot}$ star. Note that no fragmentation takes place in the inner $70 \mathrm{AU}$ of the disc, although some low-mass secondaries are subsequently scattered into the inner region. Two low-mass secondaries have already formed, and three or four more are about to form. 
liberated brown dwarfs retain discs, with masses $\lesssim 0.01 \mathrm{M}_{\odot}$ and radii $\lesssim 40 \mathrm{AU}$, (ii) brown dwarfs that remain bound to the ur star are about four times more likely to be in a tight binary system with another brown dwarf than those which are liberated, (iii) BD/BD binaries tend to have high mass ratios $(\bar{q} \sim 0.85)$, (iv) very few planemos remain bound to the ur star, but some end up in tight binary systems with brown dwarfs and (v) wide $\mathrm{BD} / \mathrm{BD}$ binaries $(a \sim 200 \mathrm{AU})$ are also formed.

\section{Conclusions}

Disc fragmentation and the subsequent eigen evolution of the resulting small- $N$ system explain (a) the brown-dwarf desert, (b) the statistics of BD/BD and BD/planemo binaries (frequency, location and orbital parameters), (c) the properties of discs around young brown dwarfs and (d) the existence of free-floating planemos. This scenario also predicts (e) the low velocity dispersion of brown dwarfs and hence their spatial distribution in clusters and (f) that planets cannot form on a dynamical timescale, even with convective cooling. In addition, disc fragmentation has the advantage that many of the statistical properties of brown dwarfs and low-mass hydrogen-burning stars are determined by the eigen evolution of a small- $N$ system formed within a single protostellar core, so they are largely independent of the environment or the overall dynamics of the parent cluster.

\section{References}

André, P., Belloche, A., Motte, F., \& Peretto, N. 2007, A\& A, 472, 519

Bate, M. R., Bonnell, I. A., \& Bromm, V. 2003, MNRAS, 339, 577

Burgasser, A. J., Kirkpatrick, J. D., \& Lowrance, P. J. 2005, AJ, 129, 2849

Burgasser, A. J., Reid, I. N., Siegler, N., Close, L., Allen, P., Lowrance, P. J., \& Gizis, J. 2007, in: B. Reipurth, D. Jewitt \& K. Keil (eds.), Protostars 83 Planets V, p. 427, Tucson: University of Arizona Press

Chabrier, G. 2003, PASP, 115, 763

Gammie, C. A. 2001, ApJ, 553, 174

Goldreich, P. \& Lynden-Bell, D. 1965, MNRAS, 130, 97

Goodwin, S. P., Whitworth, A. P., \& Ward-Thompson, D. 2004, A\&A, 414, 633

Hennebelle, P. \& Chabrier, G. 2008, ApJ, 684, 395

Hester, J. J., et al. 1996, AJ, 111, 2349

Klessen, R. \& Burkert, A. 2002, ApJS, 128, 287

Kroupa, P. 2002, Science, 295, 82

Luhman, K., Joergens, V., Lada, C., Muzerolle, J., Pascucci, I., \& White, R. 2007, in: B. Reipurth, D. Jewitt \& K. Keil (eds.), Protostars \& Planets V, p. 443, Tucson: University of Arizona Press

Padoan, P. \& Nordlund, A 2002, ApJ, 576, 870

Reipurth, B. \& Clarke, C. J. 2001, AJ, 122, 432

Stamatellos, D., Whitworth, A. P., Bisbas, T., \& Goodwin, S. P. 2007, A $\& A$ A 475, 37

Stamatellos, D., Hubber, D. A., \& Whitworth, A. P. 2007, MNRAS (Letters), 382, L30

Stamatellos, D. \& Whitworth, A. P. 2009a, MNRAS, 392, 413

Stamatellos, D. \& Whitworth, A. P. 2009b, MNRAS, in press (arXiv:0908.2247)

Toomre, A. 1964, ApJ, 139, 1217

Whitworth, A. P., Bate, M. R., Nordlund, Å., Reipurth, B., \& Zinnecker, H. 2007, in: B. Reipurth, D. Jewitt \& K. Keil (eds.), Protostars \& Planets V, p. 459, Tucson: University of Arizona Press

Whitworth, A. P. \& Stamatellos, D. 2006, $A \mathscr{E} A$ A, 458, 817

Whitworth, A. P. \& Zinnecker, H. 2004, A\& A, 427, 299 